\title{
Guided wave modes in porous cylinders: Theory
}

\author{
C. J. Wisse \\ Department of Geotechnology, Delft University of Technology, P.O. Box 5028, 2600 GA, Delft, \\ The Netherlands and Department of Applied Physics, Eindhoven University of Technology, P.O. Box 513, \\ 5600 MB, Eindhoven, The Netherlands \\ D. M. J. Smeulders \\ Department of Geotechnology, Delft University of Technology, P.O. Box 5028, 2600 GA, Delft, \\ The Netherlands \\ G. Chao \\ Department of Applied Physics, Eindhoven University of Technology, P.O. Box 513, 5600 MB, Eindhoven, \\ The Netherlands \\ M. E. H. van Dongen \\ Department of Applied Physics, Eindhoven University of Technology, P.O. Box 513, 5600 MB, Eindhoven, \\ The Netherlands
}

(Received 24 November 2006; revised 8 July 2007; accepted 9 July 2007)

\begin{abstract}
The classical theory of wave propagation in elastic cylinders is extended to poro-elastic mandrel modes. The classical theory predicts the existence of undamped L modes and damped C, I, and Z modes. These waves also appear in poro-elastic mandrels, but all of them become damped because of viscous effects. The presence of the Biot slow bulk wave in the poro-elastic material is responsible for the generation of additional mandrel modes. One of them was already discussed by Feng and Johnson, and the others can be grouped together as so-called D modes. The damping of these D modes is at least as high as the damping of the free-field slow wave. (C) 2007 Acoustical Society of America. [DOI: 10.1121/1.2767418]
\end{abstract}

PACS number(s): 43.40.At, 43.20.Mv, 43.20.Jr, 43.20.Hq [RR] Pages: 2049-2056

\section{INTRODUCTION}

For the interpretation of acoustic borehole logs a thorough understanding of the effects of rock properties on acoustic wave propagation is required. Examples of these properties are the porosity, permeability, lithology, and hydrocarbon saturation. In conventional acoustic borehole logging the first arrival of the acoustic wave train can be used to determine the porosity. The full wave train is applied to determine mechanical properties, while one of the late arrivals, the Stoneley wave, is used to obtain permeability data. ${ }^{1,2}$ The properties of the different arrivals in the time domain can be studied separately.

Rosenbaum ${ }^{3}$ used Biot's theory ${ }^{4,5}$ to describe the wave modes in a borehole. Schmitt et al., ${ }^{6}$ Liu, ${ }^{7}$ and Winkler et al. ${ }^{8}$ demonstrated the permeability dependence of the Stoneley wave. It was argued that the permeability dependence of the Stoneley wave could be explained by its relation to the slow Biot wave.

The present study is concerned with wave modes in a porous cylinder. Special attention is given to the influence of the slow Biot wave on the wave modes. The configuration is given in Fig. 1, representing a porous mandrel inside a steel tube. Between the porous cylinder and the inner wall of the tube a water-filled annulus exists. Experimental results for this configuration were discussed in a previous paper. ${ }^{9}$ In these experiments, a series of pressure waveforms is recorded at different positions along the cylinder. A FFT-Prony method is implemented to transform the data from the time- space domain to the frequency-wavenumber domain. In this way, frequency-dependent velocities and attenuation of individual mandrel modes can be studied separately. The fundamental mode is the Stoneley wave propagating along the interface between the porous medium and the liquid. However, also higher-order modes were observed in some of our previous experiments. ${ }^{10}$ This observation motivates our systematic study of these higher-order modes, which may appear in poro-elastic mandrels.

First, the wave modes in nonporous elastic mandrels are revisited. Wave propagation in elastic cylinders is described by the Pochhammer equation, which has been investigated by many researchers. ${ }^{11,12}$ In essence, this equation describes the propagation of wave modes through an elastic cylinder (mandrel) with free-surface boundary conditions. A finite number of undamped modes occur, as well as an infinite number of strongly damped modes. ${ }^{13,14}$

Gardner ${ }^{15}$ was the first to study wave modes in porous cylinders. In that work the long-wavelength approximation was combined with the low-frequency limit of Biot's theory. Two types of wave modes were predicted, one related to the free-field fast wave and one related to the free-field slow wave. Berryman ${ }^{16}$ used the full Biot theory to study the wave modes in a poro-elastic cylinder. When the pores of the cylinder have an open connection with the outside world (openpore condition), three wave modes were found that are similar to the wave modes in an elastic cylinder. No waves associated with the free-field slow wave were found. When the pores are sealed from the outside world (closed-pore con- 


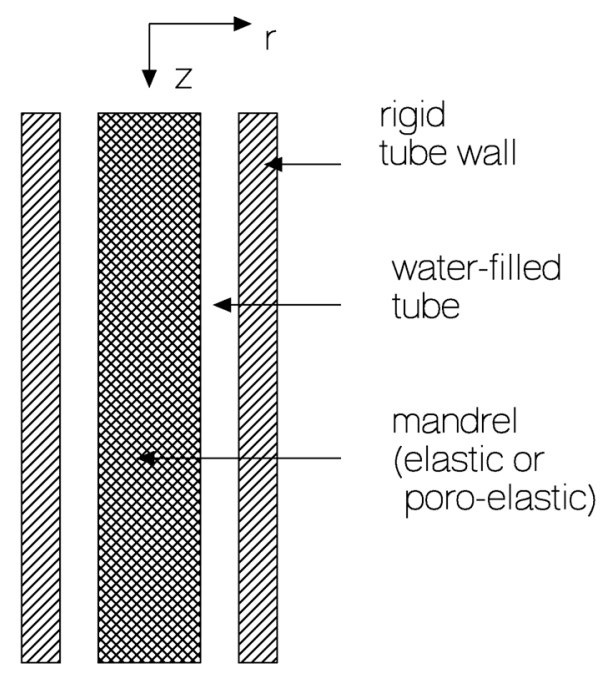

FIG. 1. (Color online) Schematic diagram for computing the characteristics of axially symmetric modes is a water-filled tube with a mandrel that may be either elastic or poro-elastic. The mandrel radius is $a$, the inner tube radius is $b$. The configuration is presumed to be of infinite length.

dition), a wave type was found that had several properties of the free-field slow wave. Its velocity, however, was lower than that of the free-field slow wave. It was suggested that this wave type is similar to the true surface wave discussed by Feng and Johnson. ${ }^{17}$ Hsu et al. ${ }^{18}$ investigated wave modes in a (porous) mandrel in a cylindrical borehole surrounded by an elastic formation. Their configuration is similar to ours. A wave mode that could only be explained by the presence of the slow Biot wave was found. Further work on porous cylinders has been carried out by Johnson and Kostek, ${ }^{19}$ who provided a comparison between the approximation of Gardner and the full Biot theory for the first mode type. Previous papers have in common that usually one or two waves are discussed that are of importance for practical purposes. In this paper, the full set of wave modes that exist in poro-elastic cylinders is discussed. In this respect, it is an extension of the work by Zemanek ${ }^{14}$ to the poro-elastic case.

The approach of this paper is as follows. First, the results for the undamped and damped modes in a nonporous elastic cylinder are briefly reviewed. Next, the computational results are given for a water-saturated mandrel made out of natural Bentheimer sandstone, which is a typical example of the class of consolidated sandstones.

\section{ELASTIC CYLINDERS}

A detailed analysis of the wave modes in an elastic cylinder is given by Onoe et al. ${ }^{13}$ and Zemanek. ${ }^{14}$ Following the approach of Miklowitz ${ }^{12}$ for the axial symmetric case, the following decomposition for the displacement vector $\mathbf{u}_{s}$ is used:

$$
\mathbf{u}_{s}=\nabla \varphi_{s}+\nabla \times \nabla \times\left(\eta_{s h} \mathbf{e}_{z}\right),
$$

where the potentials $\varphi_{s}$ and $\eta_{s h}$ satisfy the wave equation with respect to the compressional and shear wave velocity, respectively. The unit vector in the $z$-direction is $\mathbf{e}_{z}$. For cylindrical coordinates with $z$ as the axial coordinate and $r$ as the radial coordinate it can be derived that ${ }^{12}$

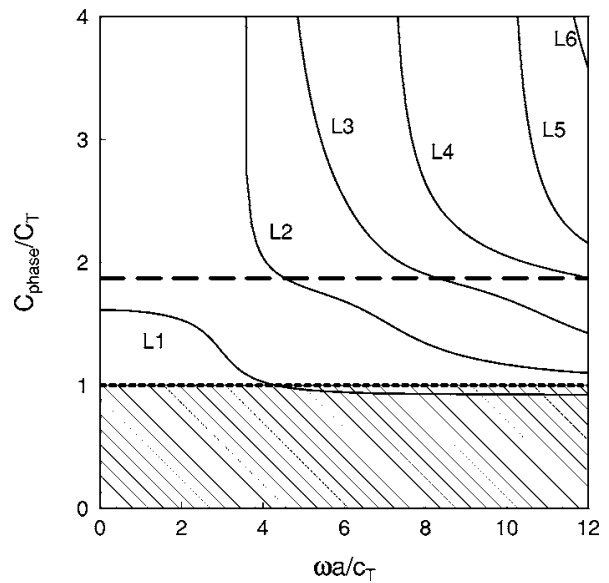

FIG. 2. Undamped modes: Frequency dependence of the phase velocity for an elastic cylinder with $\nu=0.3$. Dashed line: $\omega=C_{L} \operatorname{Re}(k)$. Shaded region: $\omega / C_{T}<\operatorname{Re}(k)$. For reference, also the free-field shear (dotted line) and compressional (dashed line) waves are shown.

$$
\varphi=A_{c 1} J_{0}\left(k_{r c 1} r\right) e^{i(\omega t-k z)}
$$

and

$$
\eta_{s h}=C_{s h} J_{0}\left(k_{r s h} r\right) e^{i(\omega t-k z)} .
$$

The radial wavenumbers $k_{r c 1}$ and $k_{r s h}$ are defined as

$$
k_{r c 1}=\sqrt{\frac{\omega^{2}}{C_{L}^{2}}-k^{2}}, \quad k_{r s h}=\sqrt{\frac{\omega^{2}}{c_{T}^{2}}-k^{2}} .
$$

In these expressions $c_{L}$ and $c_{T}$ denote the compressional and shear velocity, respectively. The free-surface condition requires that all stresses at $r=a$ vanish:

$$
\tau_{r r}=0, \quad \tau_{r z}=0 .
$$

For the amplitude coefficients the following system of equations is obtained:

$$
\underline{\underline{\mathbf{M}}}\left(A_{c 1}, C_{s h}\right)^{T}=\underline{0} \text {, }
$$

where the elements of $\underline{\underline{\mathbf{M}}}$ can be derived from the boundary conditions (see Ref. 12, pp. 220-221). The requirement that the determinant of coefficients must vanish yields the frequency equation: $F(\omega, k)=0$. This equation is the wellknown Pochhammer frequency equation. This equation is solved using a Newton-Raphson algorithm. At a given frequency $\omega$, a finite number of undamped modes and an infinite number of damped modes exist. ${ }^{14}$

The results of the calculations for the undamped modes are shown in Fig. 2. On the horizontal axis the dimensionless frequency is shown, while on the vertical axis the dimensionless phase velocity is given, where $a$ is the radius of the cylinder. Pochhammer's equation is determined uniquely by the variables $\omega a / c_{T}, k a$, and Poisson's ratio $\nu .{ }^{11}$ The results are given for $\nu=0.3$. For comparison, the free-field velocities of the compressional and the shear waves are also given. The solutions in the dashed region correspond to surface waves. In Fig. 2, one wave mode, which is propagative in the entire frequency range, is observed. This mode is labeled L1. In the low-frequency limit the phase velocity of the L1 mode is called the bar velocity. In the high-frequency limit the phase velocity is equal to the velocity of the Rayleigh wave on a 


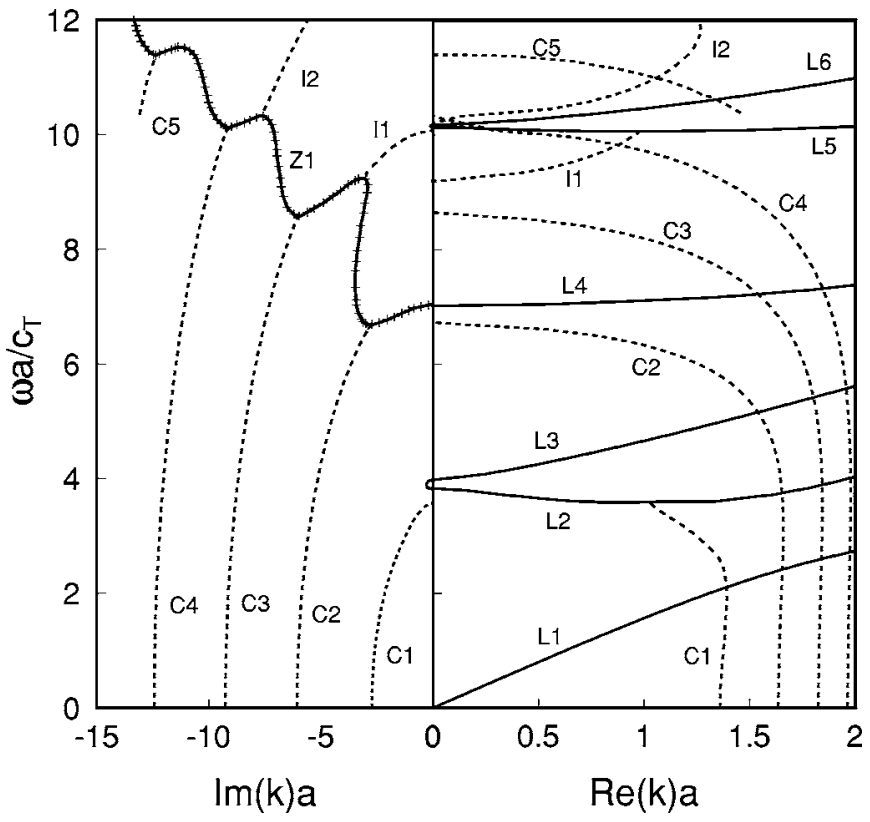

FIG. 3. Modes of an elastic cylinder in the complex plane. The solid lines correspond to the L modes of Fig. 2(a). The pairs of dashed lines correspond to the imaginary and real parts of $k$. The computations were performed for an elastic cylinder with $\nu=0.3$.

flat interface. At these high frequencies, the wavelength becomes large enough so that the curvature of the surface is negligible with respect to the wavelength. The higher-order modes L $2, \ldots$, L6 are characterized by the high-frequency limit of the phase velocity, which is the free-field shear velocity. At lower frequencies they show cutoff behavior, i.e., below the cutoff frequencies these modes are nonpropagating.

For the same cylinder, also an infinite number of damped modes exist, for which $\operatorname{Re}(k) a$ and $\operatorname{Im}(k) a$ are given in Fig. 3 as a function of the frequency $\omega a / c_{T}$. For comparison, also the undamped wave modes are drawn (solid lines). Modes that appear only on the right-hand side of Fig. 3 are propagatory and undamped. Modes that appear only on the left-hand side of Fig. 3 [the $\operatorname{Re}(k) a-\omega a / c_{T}$ part] are nonpropagative and damped, whereas modes that appear in both plots are propagating damped waves. The latter are indicated by pairs of dashed lines: one in the $\operatorname{Re}(k)$ plane, and a corresponding one in the $\operatorname{Im}(k)$ plane. Labels $\mathrm{C}$ and $\mathrm{I}$ are used here. The $\mathrm{C}$ modes exist at zero frequency and for higher frequencies they catch up to the $\mathrm{Z} 1$ mode, which is a nonpropagative mode. The I modes originate from the $\mathrm{Z} 1$ mode and eventually catch up to the nonpropagative $\mathrm{Z} 2$ mode (not shown in Fig. 3). The C, I, and Z modes play an important role near the ends of cylinders with finite length, ${ }^{14}$ but the discussion of end aspects is beyond the scope of the present paper.

\section{POROUS CYLINDER IN THE SHOCK TUBE}

The configuration of the liquid-loaded water-saturated porous cylinder is given in Fig. 1. The cylinder is considered infinitely long. The radius of the porous cylinder is denoted by $a$, while the inner radius of the surrounding tube is denoted by $b$. Biot's theory is used to describe wave propaga-
TABLE I. Parameter values.

\begin{tabular}{lc}
\hline \hline Density of water $\rho_{w}\left(\mathrm{~kg} / \mathrm{m}^{3}\right)$ & 998 \\
Dynamic viscosity of water $\eta(\mathrm{Pa} \mathrm{s})$ & $10^{-3}$ \\
Water bulk modulus $K_{f}(\mathrm{GPa})$ & 2.2 \\
Density of the solid $\rho_{s}\left(10^{3} \mathrm{~kg} / \mathrm{m}^{3}\right)$ & 2.62 \\
Porosity $(\%)$ & 0.22 \\
Permeability $k_{0}\left(10^{-12} \mathrm{~m}^{2}\right)$ & 2.73 \\
Tortuosity & 2.9 \\
Constrained modulus $K_{p}(\mathrm{GPa})$ & 17 \\
Shear modulus $\mathrm{G}(\mathrm{GPa})$ & 8.0 \\
\hline \hline
\end{tabular}

tion in the porous cylinder. A potential formulation based on Biot's theory is used to solve the wave equation in cylindrical coordinates in the frequency domain. This formulation takes into account the two free-field compressional waves and the free-field shear wave in the porous cylinder as well as the compressional wave that propagates in the fluid. The boundary conditions lead to a linear system for the amplitudes of the waves. In order to find the wave modes the determinant of this system must be equal to zero. This condition leads to a secular equation for the wavenumber of the guided wave. The secular equation is solved using a zero search routine based on Newton-Raphson's iteration method in the complex plane. At $r=a$ the open-pore boundary conditions as given by Deresiewicz and Skalak $^{20}$ and Rosenbaum $^{3}$ are applied, while at $r=b$ the radial displacement of the water is assumed to be zero. In our experiments, this is a realistic assumption indeed, as the walls of the shock tube are made of 25-mm-thick stainless steel. Hence, the following boundary conditions apply:

(1) at $r=a: \sigma_{r r}=0$,

(2) at $r=a: \sigma_{r z}=0$,

(3) at $r=a:(1-\phi) u_{s r}+\phi u_{f r}=u_{r w}$,

(4) at $r=a: p=p_{w}$,

(5) at $r=b: u_{r 2}=0$.

Using the expressions for the displacements and stresses as given in Appendix A, the five unknown amplitudes can be written as

$$
\underline{\underline{\mathbf{M}}}\left(A_{c 1}, \quad A_{c 2}, \quad C_{s h}, \quad B_{w}, \quad A_{w}\right)^{T}=\underline{0} .
$$

The elements of matrix $\underline{\underline{\mathbf{M}}}$ are given in Appendix B.

\section{COMPUTATIONAL RESULTS FOR PORO-ELASTIC CYLINDERS}

The computations are performed for a water-saturated Bentheim sandstone whose properties are given in Table I. This is a fast formation, i.e., the shear velocity is higher than the water velocity. The computations were performed with a diameter of the cylinder of $70 \mathrm{~mm}$, while the inner diameter of the tube is $77 \mathrm{~mm}$, i.e., $a=0.909 b$ (see Fig. 1).

Some of the modes that were found show similarities with the L, C, and I modes of the nonporous elastic cylinders examined in Sec. III, and consequently these modes are labelled L, C, and I here as well. Furthermore, a surface mode is found, which will be denoted by S. Due to the presence of the free-field Biot slow wave in the porous cylinder, an extra class of modes occurs, which is denoted by D. Because of 

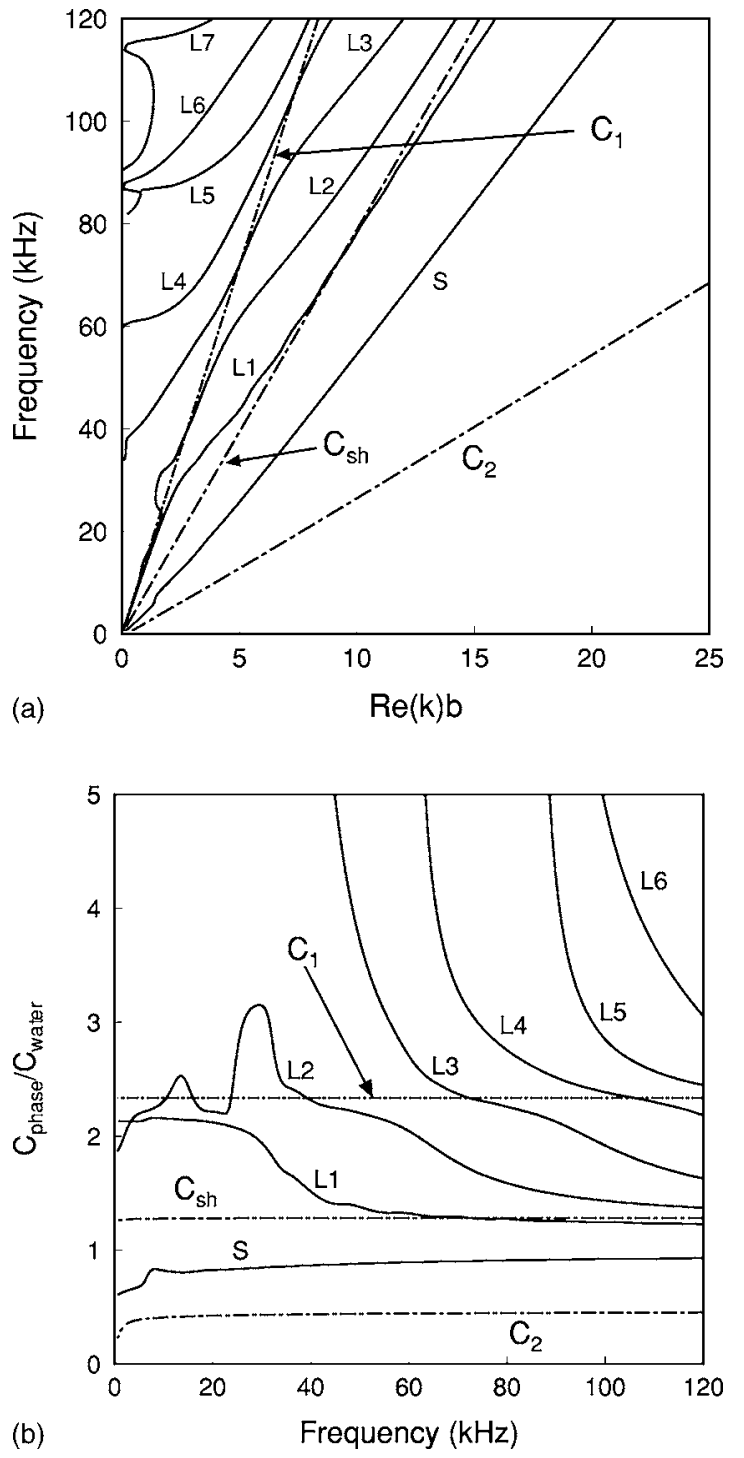

FIG. 4. Frequency dependence of $\operatorname{Re}(k)$ (a) and the phase velocity (b) for the $\mathrm{L}$ and $\mathrm{S}$ modes. The wavenumber is normalized by the inner radius of the shock tube $b$, the phase velocity by the sound velocity of water. The free-field solutions for the fast $\left(\mathrm{C}_{1}\right)$, the slow $\left(\mathrm{C}_{2}\right)$, and the shear wave $\left(\mathrm{C}_{s h}\right)$ are also given. The computations are performed for a Bentheimer sandstone cylinder with a diameter of $70.0 \mathrm{~mm}$. Note that the full computational results are presented in three figures. $\mathrm{L}$ and $\mathrm{S}$ modes are presented here, the $\mathrm{C}$ and I modes are shown in Fig. 7, and the D modes are shown in Fig. 8.

the complexity of the complete mode pattern the results are presented in separate figures. First, the $\mathrm{L}$ and $\mathrm{S}$ modes will be discussed, which are characterized by a relatively low damping factor. These results are shown in Fig. 4, while the results for the C, I, and D modes are shown in Figs. 7 and 8.

\section{A. $L$ and $S$ modes}

The data for the $\mathrm{L}$ and $\mathrm{S}$ modes are presented in the same way as for the nonporous elastic case, but, for practical purposes, ${ }^{9}$ the frequency instead of the dimensionless parameter $\omega a / c_{T}$ is used. In Fig. 4(a) the frequency versus the real part of $k$ is plotted, while in Fig. 4(b) the frequency dependence of the phase velocities is given.

Note that $\operatorname{Re}(k)$ is normalized by the inner radius of the shock tube $b$, while the phase velocities are normalized by the sound velocity of water. For comparison, also the freefield solutions for the fast $\left(C_{1}\right)$, slow $\left(C_{2}\right)$, and shear wave $\left(C_{s h}\right)$ are given.

The L1 and L2 modes have a finite phase velocity over the entire frequency range, while the higher-order L modes are cut off below a certain frequency. The L modes in Fig. 4 show strong similarities with the results of Fig. 2, where one mode that is propagative over the entire frequency range is observed, while the other modes were cut off below a critical frequency. There is, however, an important difference with respect to the case of nonporous elastic cylinders. The L1 mode for the nonporous elastic cylinder is undamped over the entire frequency range, and the higher-order $\mathrm{L}$ modes are undamped above the cutoff frequency. For the porous material, however, the free-field fast, slow, and shear waves are damped, and, consequently, all the L modes are damped over the entire frequency range. The damping coefficients of the L1 mode over the entire frequency range, and of the higherorder L modes above the cutoff frequency, are low, i.e., $|\operatorname{Im}(k) b| \ll 1$ (not plotted). The high-frequency velocity of the L1 mode equals the sound speed of water and the damping tends to zero. This wave is therefore associated with wave motion in the annulus. In the low-frequency limit, the phase velocity is somewhat lower than the free-field velocity of the fast wave. For the purely elastic case, this limiting speed was the so-called bar velocity $\sqrt{E / \rho}$. Further analysis showed that for this mode radial and axial stress components are of equal importance at high frequencies. ${ }^{21}$ In the lowfrequency limit the one-dimensional stress situation is obtained, i.e., the axial stress is dominant over the radial and shear stresses, and pore pressures are of the same order as the pressure in the annulus.

In the high-frequency limit, the L2 phase velocity reaches the phase velocity of the free-field shear velocity. At low frequencies the phase velocity remains finite, which is different from the behavior of the L2 mode for the nonporous elastic case. The phase velocity of the latter mode becomes infinite at the cutoff frequency. Note also that the damping of the $\mathrm{L} 2$ mode for the porous cylinder is relatively large at low frequencies $(|\operatorname{Im}(k) b|>1)$ (see Fig. 7).

Now the $\mathrm{S}$ mode is further analyzed. This wave is generally conspicuously present in our experiments ${ }^{9}$ and therefore merits further investigation. Figure 5 displays the frequency dependence of the phase velocity and the damping coefficient of the $\mathrm{S}$ mode in the $1-1000-\mathrm{kHz}$ frequency range. For comparison the pseudo-Stoneley wave (denoted $\mathrm{PS}_{41}$ in the figure) for a plane interface is also shown. ${ }^{21}$ From Fig. 5 it is observed that the $\mathrm{S}$ mode is equivalent to the plane-interface pseudo-Stoneley wave in the high-frequency range, where the wavelengths involved are much shorter than the radii of the shock tube and the porous cylinder. Both the phase velocities and the damping coefficients are nearly identical. At lower frequencies, the S mode is influenced by the curvatures of the porous cylinder and of the wall of the shock tube, as the wavelength becomes of the order of magnitude of the radii of the shock tube and the porous cylinder.

Figure 6 shows the radial distributions of the stresses and pressures for the $\mathrm{S}$ mode at $120 \mathrm{kHz}$. The stresses and pressures are normalized by the pore pressures at the radial 


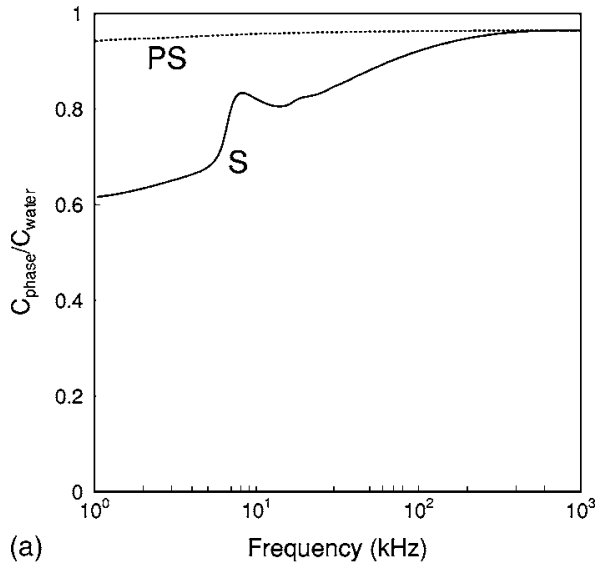

(a)

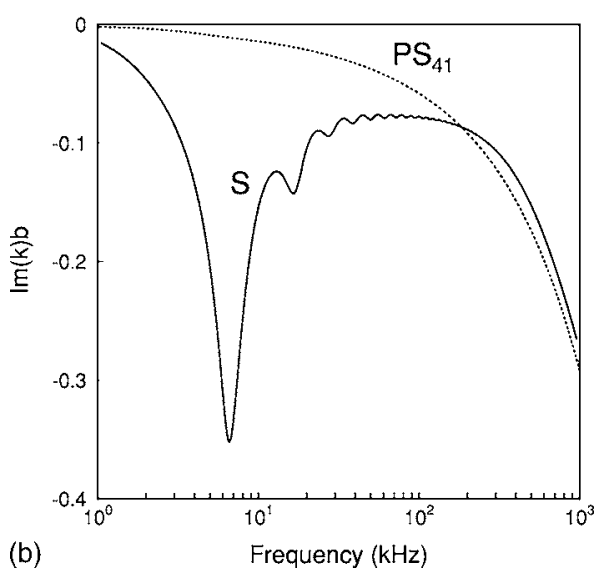

FIG. 5. Frequency dependence of the phase velocity (a) and damping (b) for the $\mathrm{S}$ mode in the $1-1000-\mathrm{kHz}$ frequency range. The results for the pseudoStoneley wave for a flat interface are also shown. The computations are performed for a Bentheimer sandstone cylinder with a diameter of $70.0 \mathrm{~mm}$. Parameter values are given in Table I.

surface of the porous cylinder $r=a$. We notice in Fig. 6 that the typical behavior of the surface wave is present at $120 \mathrm{kHz}$ : a pressure peak at the wall is observed, followed by an exponential-like decay. The pressure oscillations for $r / b$ values below 0.4 are generated by the free-field slow wave. We found that as the frequency is lowered, the pressure peak at the wall becomes broader, and the surface-wave character disappears. It can be argued that the $\mathrm{S}$ mode is associated with a surface wave at high frequencies, while it corresponds to a bulk wave type at low frequencies. Note that this is similar to the corresponding mode in a borehole, which is associated with the so-called "water-hammer" at low frequencies and with the pseudo-Stoneley wave at high frequencies. Furthermore, it can be remarked that over the entire frequency range, the annulus pressures are of the same order as the pore pressures in the porous cylinder.

Summarizing, from the analysis of the S, L1, and L2 modes, it can be stated that for the S and L1 modes the motion of the water in the annulus plays an important role. The annulus pressures for these modes are of the same order of magnitude as the stresses and pressures in the porous cylinder. The L2 mode, however, is dominated by the motion of the solid matrix. The same behavior can be expected for the higher-order wave modes. It is worthwhile to mention that in
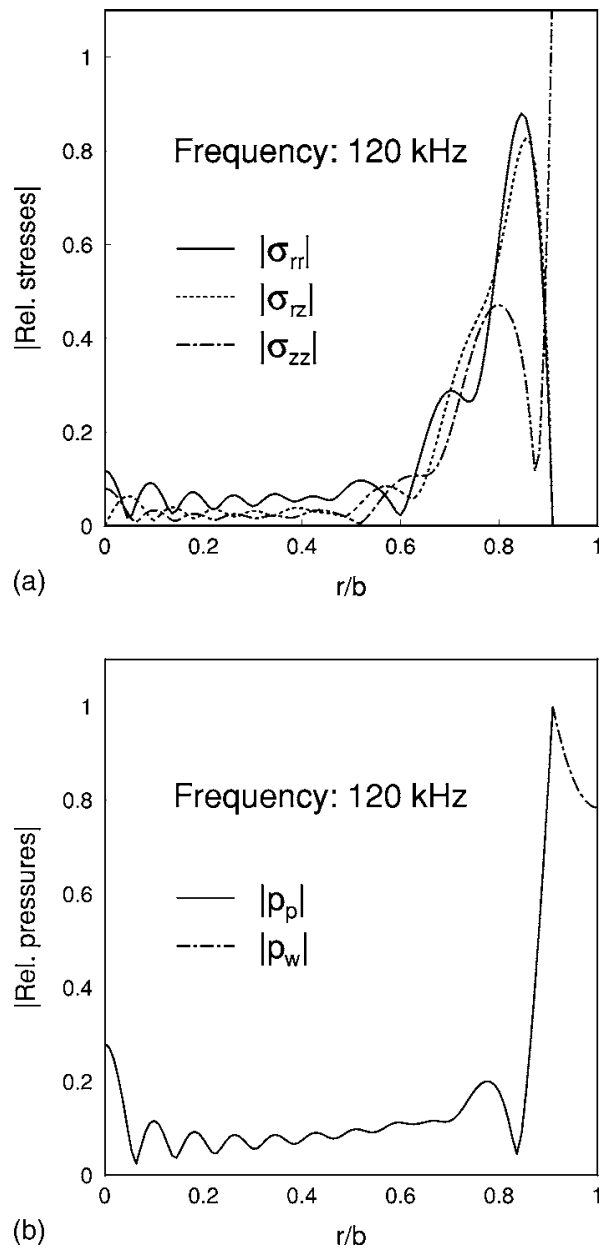

FIG. 6. Radial distributions of the stresses (a) and pressures (b) for the $\mathrm{S}$ mode at $120 \mathrm{kHz}$. Both the stresses and pressures were normalized by $\left.p_{p}\right|_{r=a}$. The calculations were performed for a Bentheim cylinder with a diameter of $70.0 \mathrm{~mm}(a=0.909 b)$. The pores at the boundary were open.

some of our previous experiments we observed the excitation of $\mathrm{L}$ modes in a borehole configuration as well. ${ }^{10}$

\section{B. C and I modes}

Our numerical computations also predict a number of strongly damped modes, which are similar to the damped $\mathrm{C}$ and I modes that we observed in computing the nonporous elastic case (see Fig. 3). Consequently, these modes for the poro-elastic cylinder are labelled $\mathrm{C}$ and I as well. In Fig. 7, the results are presented in the same way as for the nonporous elastic case. The $\mathrm{C}$ modes are indicated by dashed lines, and the I modes are indicated by solid lines. For comparison, some of the L modes that were already presented in Fig. 4 are plotted again. The behavior of $\operatorname{Re}(k)$ of the $\mathrm{C}$ modes for the porous cylinder is somewhat different from the $\mathrm{C}$ modes for the elastic case, which have a finite value of $\operatorname{Re}(k)$ at low frequencies. For the $\mathrm{C}$ modes of the porous cylinder, $\operatorname{Re}(k)$ is zero in the low-frequency limit. The $\mathrm{C}$ modes are connected to the I modes via modes in the third quadrant of the $k$ plane. Those modes have infinite amplitudes for $z \rightarrow \infty$, and are not shown in Fig. 7. 


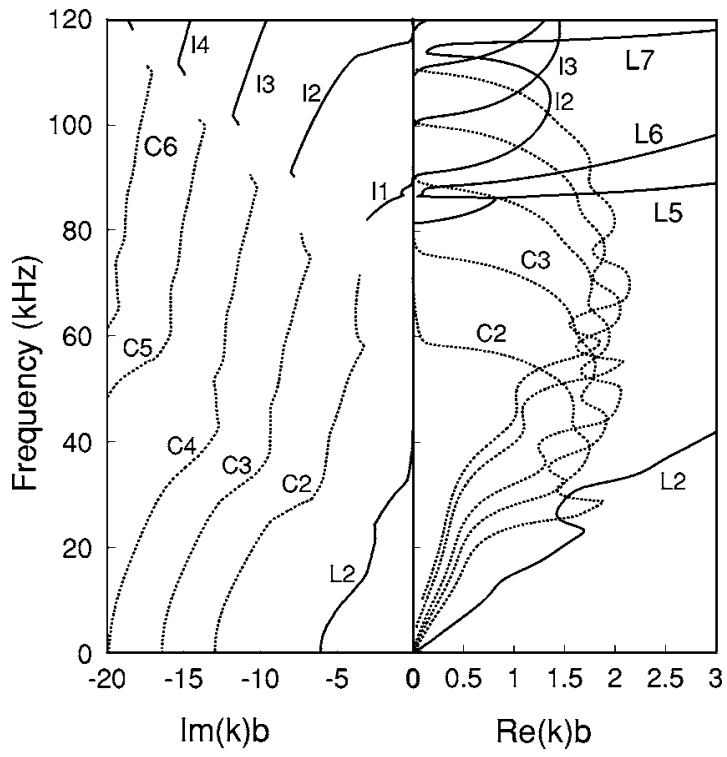

FIG. 7. Frequency dependence of $\operatorname{Im}(k)$ and $\operatorname{Re}(k)$ for the $\mathrm{C}$ and $\operatorname{I}$ modes. As a reference some $\mathrm{L}$ modes are given. The wavenumber is normalized by the inner radius of the shock tube $b$. The computations were performed for a Bentheim cylinder with a diameter of $70.0 \mathrm{~mm}$. The pores at the boundary were open. Note that the full computational results are presented in three figures. The $\mathrm{L}$ and $\mathrm{S}$ modes are shown in Fig. 4, while the $\mathrm{D}$ modes are shown in Fig. 8.

\section{D modes}

Up to now it was possible to relate our computational results to the nonporous elastic cylinder. For the poro-elastic cylinder in the shock tube, however, an extra mode type has been found, closely related to the existence of the free-field slow wave. The occurrence of this wave mode family for open-pore boundary conditions is in agreement with the predictions by Gardner, ${ }^{15}$ Liu, ${ }^{7}$ and Hsu et al. ${ }^{18}$ In the following, the slow wave mode is denoted by D. In fact, this mode is essentially a slow wave with some coupling to the other mode types. The results for the D modes are shown in Fig. 8, where the frequency dependence of the real and imaginary parts of $k$ is shown. In this figure the dashed lines indicate the free-field solution for the slow wave. From these results it is clearly observed that the D modes have the free-field solution of the slow wave as their high-frequency limit. The damping of these modes is at least as high as the damping of the slow wave and of the same order of magnitude as the damping of the $\mathrm{C}$ modes.

The pore pressures and the stress components are of the same order of magnitude, while the pressures in the center of the cylinder are much larger than the gap pressure. This means that the wave motion in the gap is of less importance for the D modes. ${ }^{21}$

\section{CONCLUSIONS}

Porous mandrels are investigated. The so-called L modes are equivalent to the ones in a nonporous elastic cylinder. These modes are characterized by cutoff frequencies and their phase velocities in the high-frequency limit, which are equal to the shear velocity. For the porous cylinder, an equivalent yet strongly damped set of modes is found. Fur-

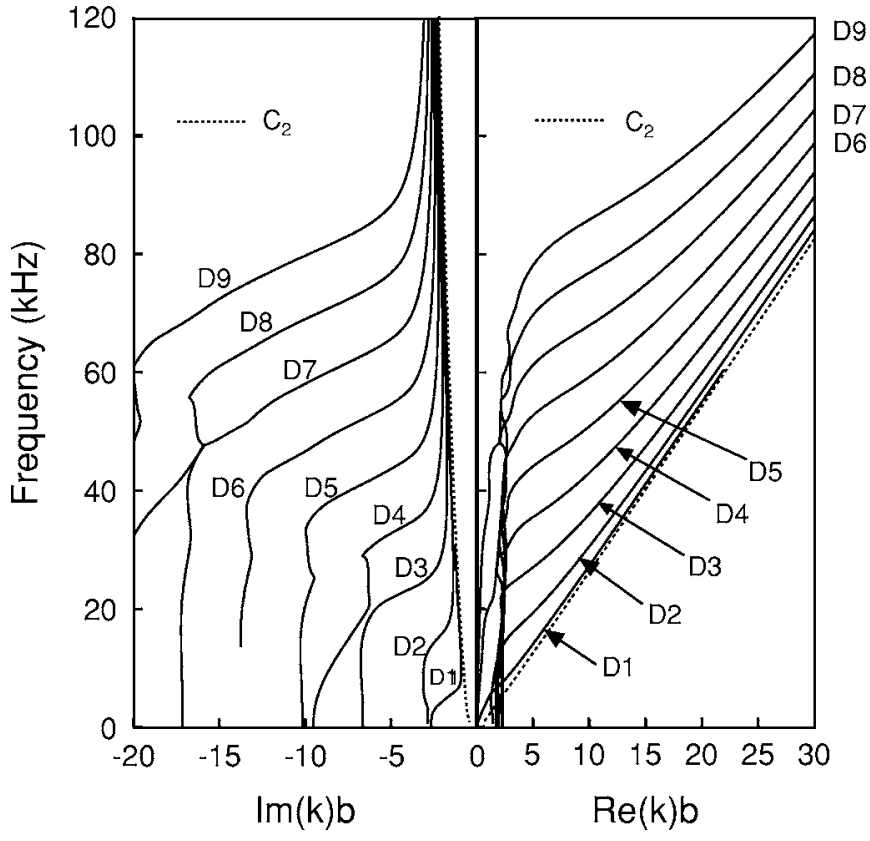

FIG. 8. Frequency dependence of $\operatorname{Im}(k)$ and $\operatorname{Re}(k)$ for the $\mathrm{D}$ modes. The wavenumber is normalized by the inner radius of the shock tube $b$. The computations were performed for a Bentheimer sandstone cylinder with a diameter of $70.0 \mathrm{~mm}$. The pores at the boundary were open. The free-field solution $C_{2}$ for the slow wave is indicated by the dashed line. Note that the full computational results are presented in three figures. The $\mathrm{L}$ and $\mathrm{S}$ modes are shown in Fig. 4, while the $\mathrm{C}$ and I modes are shown in Fig. 7.

thermore, a surface mode $\mathrm{S}$ occurs at the interface between a porous cylinder and a relatively large water-filled gap. At high frequencies this wave corresponds to the pseudoStoneley wave. Extra bulk wave modes, the D modes, occur due to the presence of the Biot slow wave. The damping of the higher-order D modes is at least as high as the damping of the Biot slow wave itself.

\section{APPENDIX A: BIOT THEORY IN POROUS CYLINDERS}

The potential $\varphi_{s}$ in Eq. (1) consists of two parts, one for the fast Biot wave and one for the slow Biot wave: ${ }^{16,22}$

$$
\varphi_{s}=\varphi_{s c 1}+\varphi_{s c 2} .
$$

The fast wave potential satisfies the wave equation with respect to the complex-valued velocity of the fast wave $c_{1}$ and the slow wave potential satisfies the wave equation with respect to the complex-valued velocity of the slow wave $c_{2}$. The shear potential $\eta_{s h}$ satisfies the wave equation with respect to the complex-valued velocity of the shear wave $c_{s h}$. For $\varphi_{s c 1}, \varphi_{s c 2}$, and $\eta_{s h}$ it is possible to derive that

$$
\begin{aligned}
& \varphi_{s c 1}=A_{c 1} J_{0}\left(k_{r c 1} r\right) e^{i(\omega t-k z)}, \\
& \varphi_{s c 2}=A_{c 2} J_{0}\left(k_{r c 2} r\right) e^{i(\omega t-k z)},
\end{aligned}
$$

and

$$
\eta_{s h}=C_{s h} J_{0}\left(k_{r s h} r\right) e^{i(\omega t-k z)} .
$$

The radial wavenumbers are defined by

$$
k_{r c 1}=\sqrt{\frac{\omega^{2}}{c_{1}^{2}}-k^{2}},
$$




$$
\begin{aligned}
& k_{r c 2}=\sqrt{\frac{\omega^{2}}{c_{2}^{2}}-k^{2}}, \\
& k_{r s h}=\sqrt{\frac{\omega^{2}}{c_{s h}^{2}}-k^{2}},
\end{aligned}
$$

with $\operatorname{Im}\left(k_{r c 1}\right) \leq 0, \operatorname{Im}\left(k_{r c 2}\right) \leq 0$, and $\operatorname{Im}\left(k_{r s h}\right) \leq 0$. The potentials for the fluid are related to the potentials of the solid by the following expressions:

$$
\varphi_{f c 1}=\beta_{c 1} \varphi_{s c 1}, \quad \varphi_{f c 2}=\beta_{c 2} \varphi_{s c 2}, \quad \eta_{f s h}=\beta_{s h} \eta_{s h} .
$$

The coefficients $\beta_{c 1}, \beta_{c 2}$, and $\beta_{s h}$ are frequency-dependent and can be derived from the equations of motion (see Ref. 22, p. 131).

The displacements of the solid and the fluid can be derived from the potentials by the following relations:

$$
u_{s r}=\frac{\partial \varphi_{s c 1}}{\partial r}+\frac{\partial \varphi_{s c 2}}{\partial r}+\frac{\partial^{2} \eta_{s h}}{\partial z \partial r}
$$

and

$$
u_{f r}=\beta_{c 1} \frac{\partial \varphi_{s c 1}}{\partial r}+\beta_{c 2} \frac{\partial \varphi_{s c 2}}{\partial r}+\beta_{s h} \frac{\partial^{2} \eta_{s h}}{\partial z \partial r} .
$$

For the isotropic case the stress-strain relations are given by Biot. $^{23}$ Using cylindrical coordinates the radial stress component acting on the solid part can be written as

$$
\tau_{r r}=(P-2 G) \nabla^{2} \varphi_{s}+Q \nabla^{2} \varphi_{f}+2 G\left[\frac{\partial^{2} \varphi_{s}}{\partial r^{2}}-\frac{\partial^{3} \eta_{s h}}{\partial z \partial r^{2}}\right],
$$

where the elasticity coefficients $P, Q, R$, and $G$ have been introduced. The relation between these coefficients and measurable quantities are given by Allard. ${ }^{22}$ For the shear stress $\tau_{r z}$ it follows that

$$
\tau_{z r}=G\left\{2 \frac{\partial^{2} \varphi_{s}}{\partial z \partial r}+\frac{\partial^{3} \eta_{s h}}{\partial z^{2} \partial r}-\frac{\partial}{\partial r}\left[\frac{1}{r} \frac{\partial}{\partial r}\left(r \frac{\partial \eta_{s h}}{\partial r}\right)\right]\right\} .
$$

The stress component acting on the fluid part can be written as

$$
\tau=Q \nabla^{2} \varphi_{s}+R \nabla^{2} \varphi_{f}
$$

The radial stress acting on the solid part can be related to the radial component of the so-called intergranular stress $\sigma_{r r}$ and the pore pressure $p_{p}$ by

$$
\tau_{r r}=-\sigma_{r r}-(1-\phi) p_{p},
$$

where $\phi$ is the porosity. For $\sigma_{r r}$ it holds that

$$
\sigma_{r r}=-\Lambda_{c 1} \nabla^{2} \varphi_{s c 1}-\Lambda_{c 2} \nabla^{2} \varphi_{s c 2}-2 G\left[\frac{\partial^{2} \varphi_{s}}{\partial r^{2}}-\frac{\partial^{3} \eta_{s h}}{\partial z \partial r^{2}}\right],
$$

where

$$
\Lambda_{c 1}=P-2 G-\frac{(1-\phi) Q}{\phi}+Q \beta_{c 1}-\frac{(1-\phi) R \beta_{c 1}}{\phi}
$$

and

$$
\Lambda_{c 2}=P-2 G-\frac{(1-\phi) Q}{\phi}+Q \beta_{c 2}-\frac{(1-\phi) R \beta_{c 2}}{\phi} .
$$

For intergranular shear stress it is clear that $\sigma_{r z}=\tau_{r z}$. The pore pressure can be written as $p_{p}=-\tau / \phi$.

The wave motion in the water-filled gap is described by the wave equation of water. Hence, it is possible to derive the potential of the water-filled gap:

$$
\varphi_{w}=\left[B_{2} Y_{0}\left(k_{r w} r\right)+A_{2} J_{0}\left(k_{r w} r\right)\right] e^{i(\omega t-k z)},
$$

where the Bessel function of the second kind $Y_{0}$ is included and

$$
k_{r w}=\sqrt{\frac{\omega^{2}}{c_{w}^{2}}-k^{2}}
$$

with $\operatorname{Im}\left(k_{r w}\right) \leq 0$. The pressure in the liquid can be written as

$$
p_{w}=\rho_{w} \omega^{2} \varphi_{w}
$$

and the radial water displacement as

$$
u_{w r}=\frac{\partial \varphi_{w}}{\partial r}=-k_{r w} B_{w} Y_{1}\left(k_{r w} r\right)-k_{r w} A_{w} J_{1}\left(k_{r w} r\right),
$$

where the $e^{i(\omega t-k z)}$ dependence has been omitted.

\section{APPENDIX B: MATRIX ELEMENTS FOR THE POROUS CYLINDER}

The boundary conditions for the porous cylinder in the shock tube give the following system of equations for the amplitudes of the potentials:

$$
\underline{\underline{\mathbf{M}}}\left(A_{c 1}, \quad A_{c 2}, \quad C_{s h}, \quad B_{w}, \quad A_{w}\right)^{T}=0 .
$$

The elements of matrix $\underline{\underline{\mathbf{M}}}$ are given by

$$
\begin{aligned}
M(1,1)= & \Lambda_{c 1}\left(\frac{\omega^{2}}{c_{1}^{2}}\right) J_{0}\left(k_{r c 1} a\right)+2 G\left[k _ { r c 1 } \left(k_{r c 1} J_{0}\left(k_{r c 1} a\right)\right.\right. \\
& \left.\left.-\frac{1}{a} J_{1}\left(k_{r c 1} a\right)\right)\right], \\
M(1,2)= & \Lambda_{c 2}\left(\frac{\omega^{2}}{c_{2}^{2}}\right) J_{0}\left(k_{r c 2} a\right)+2 G\left[k _ { r c 2 } \left(k_{r c 2} J_{0}\left(k_{r c 2} a\right)\right.\right. \\
& \left.\left.-\frac{1}{a} J_{1}\left(k_{r c 2} a\right)\right)\right], \\
M(1,3)= & -2 G i k k_{r s h}\left(k_{r s h} J_{0}\left(k_{r s h} a\right)-\frac{1}{a} J_{1}\left(k_{r s h} a\right)\right),
\end{aligned}
$$

$M(1,4)=0$,

$M(1,5)=0$,

$M(2,1)=-2 i k k_{r c 1} J_{1}\left(k_{r c 1} a\right)$,

$M(2,2)=-2 i k k_{r c 2} J_{1}\left(k_{r c 2} a\right)$,

$M(2,3)=-k_{r s h}\left(k^{2}-k_{r s h}^{2}\right) J_{1}\left(k_{r s h} a\right)$,

$M(2,4)=0$, 


$$
\begin{aligned}
& M(2,5)=0, \\
& M(3,1)=\left((\phi-1)-\beta_{c 1} \phi\right) k_{r c 1} J_{1}\left(k_{r c 1} a\right), \\
& M(3,2)=\left((\phi-1)-\beta_{c 2} \phi\right) k_{r c 2} J_{1}\left(k_{r c 2} a\right), \\
& M(3,3)=\left((1-\phi)+\beta_{s h} \phi\right) i k k_{r s h} J_{1}\left(k_{r s h} a\right), \\
& M(3,4)=k_{r w} Y_{1}\left(k_{r w} a\right), \\
& M(3,5)=k_{r w} J_{1}\left(k_{r w} a\right), \\
& M(4,1)=\frac{1}{\phi}\left(\frac{\omega^{2}}{c_{1}^{2}}\right) J_{0}\left(k_{r c 1} a\right)\left(Q+R \beta_{c 1}\right), \\
& M(4,2)=\frac{1}{\phi}\left(\frac{\omega^{2}}{c_{2}^{2}}\right) J_{0}\left(k_{r c 2} a\right)\left(Q+R \beta_{c 2}\right), \\
& M(5,2)=0, \\
& M(5,3)=0, \\
& M(4,3)=0, \\
& M(4,5)=-\rho_{w} \omega^{2} J_{0}\left(k_{r w} a\right), \\
& M(5,1)=0, \\
& M(5)=-k_{r w} J_{1}\left(k_{r w} b\right) . \\
& M\left(Y_{1}^{2} Y_{0}\left(k_{r w} a\right),\right. \\
& M\left(k_{r w} b\right), \\
& M(5),
\end{aligned}
$$

The matrix elements only have combinations of $J_{0}\left(k_{r j} r\right)$, $k_{r j}^{2} J_{0}\left(k_{r j} r\right)$, and $k_{r j} J_{1}\left(k_{r j} r\right)$, where $j=c 1, c 2, s h$. These combinations are even functions of the corresponding radial wavenumbers and therefore the choice of the Riemann sheet for the square root is arbitrary. The Neumann functions $Y_{0}(x)$ and $Y_{1}(x)$ are neither an odd nor an even function of $x$. However, $Y_{0}(-x)$ can be written as the sum of $Y_{0}(x)$ and $J_{0}(x){ }^{24}$ Hence, the final result of the potential $\varphi_{w}$ as given by Eq. (A16) is not influenced by the sign of $k_{r w}$, and the choice of the Riemann sheet for the radial wavenumber $k_{r w}$ is arbitrary. The only relevant cut is the branch cut of the functions $Y_{0}$ and $Y_{1}:\left|\arg \left(k_{r w} r\right)\right|=\pi$. Passing this cut can be avoided by using $\operatorname{Im}\left(k_{r w}\right) \leq 0$.
${ }^{1}$ F. L. Paillet and C. H. Cheng. Acoustic Waves in Boreholes-The Theory and Application of Acoustic Full-Waveform Logs (CRC, Boca Raton, FL, 1991)

${ }^{2}$ X. M. Tang and C. H. Cheng, "Fast inversion of formation permeability from Stoneley wave logs using a simplified Biot-Rosenbaum model," Geophysics 61, 639-645 (1996).

${ }^{3}$ J. H. Rosenbaum, "Synthetic microseismograms: Logging in porous formations," Geophysics 39, 14-32 (1974).

${ }^{4} \mathrm{M}$. A. Biot, "Theory of propagation of elastic waves in a fluid-saturated porous solid I. Low-frequency range," J. Acoust. Soc. Am. 28, 168-178 (1956).

${ }^{5}$ M. A. Biot, "Theory of propagation of elastic waves in a fluid-saturated porous solid II. Higher frequency range," J. Acoust. Soc. Am. 28, 179-191 (1956).

${ }^{6}$ D. P. Schmitt, M. Bouchon, and G. Bonnet, "Full-wave synthetic acoustic $\operatorname{logs}$ in radially semi-infinite saturated porous media," Geophysics 53, 807-823 (1988).

${ }^{7}$ H. L. Liu, "Borehole modes in a cylindrical fluid-saturated permeable medium," J. Acoust. Soc. Am. 84, 424-431 (1988).

${ }^{8}$ K. W. Winkler, H.-S. Liu, and D. L. Johnson, "Permeability and borehole Stoneley waves: Comparison between experiment and theory," Geophysics 54, 66-75 (1989).

${ }^{9}$ C. J. Wisse, D. M. J. Smeulders, M. E. H. van Dongen, and G. Chao, "Guided wave modes in porous cylinders: Experimental results," J. Acoust. Soc. Am. 112(3), 890-895 (2002).

${ }^{10}$ G. Chao, D. M. J. Smeulders, and M. E. H. van Dongen, "Measurements of shock-induced guided and surface acoustic waves along boreholes in poroelastic materials," J. Appl. Phys. 99(9), 094904 (2006).

${ }^{11} \mathrm{~J}$. D. Achenbach, Wave Propagation in Elastic Solids (North-Holland, Amsterdam, 1973).

${ }^{12} \mathrm{~J}$. Miklowitz, The Theory of Elastic Waves and Waveguides (NorthHolland, Amsterdam, 1978).

${ }^{13}$ M. Onoe, H. D. McNiven, and R. D. Mindlin, "Dispersion of axially symmetric waves in elastic rods," J. Appl. Mech. 29, 729-734 (1962).

${ }^{14} \mathrm{~J}$. Zemanek, "An experimental and theoretical investigation of elastic wave propagation in a cylinder," J. Acoust. Soc. Am. 51, 265-283 (1971).

${ }^{15}$ G. H. F. Gardner, "Extensional waves in fluid-saturated porous cylinders," J. Acoust. Soc. Am. 34, 36-40 (1962).

${ }^{16}$ J. G. Berryman, "Dispersion of extensional waves in fluid-saturated porous cylinders at ultrasonic frequencies," J. Acoust. Soc. Am. 74, 18051812 (1983).

${ }^{17}$ S. Feng and D. L. Johnson, "High-frequency acoustic properties of a fluid/ porous solid interface," J. Acoust. Soc. Am. 74, 906-924 (1983).

${ }^{18}$ C.-J. Hsu, S. Kostek, and D. L. Johnson, "Tube waves and mandrel modes: Experiment and theory," J. Acoust. Soc. Am. 102, 3277-3289 (1997).

${ }^{19}$ D. L. Johnson and S. Kostek, "A limitation of the Biot-Gardner theory of extensional waves in fluid-saturated porous cylinders," J. Acoust. Soc. Am. 97, 741-744 (1995).

${ }^{20} \mathrm{H}$. Deresiewicz and R. Skalak, "On uniqueness in dynamic poroelasticity," Bull. Seismol. Soc. Am. 53, 783-788 (1963).

${ }^{21} \mathrm{C}$. J. Wisse, "On frequency dependence of acoustic waves in porous cylinders," Ph.D. thesis, Delft University of Technology, 1999.

${ }^{22}$ J. F. Allard, Propagation of Sound in Porous Media (Elsevier Science, Amsterdam, 1993).

${ }^{23} \mathrm{M}$. A. Biot, "Theory of elasticity and consolidation for a porous anisotropic solid," J. Appl. Phys. 26, 182-185 (1955).

${ }^{24}$ M. Abramowitz and I. A. Stegun, Handbook of Mathematical Functions (Dover, New York, 1964). 